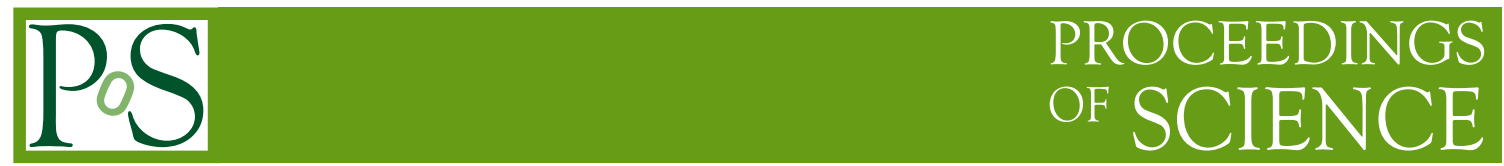

\title{
Searches for Supersymmetry
}

\section{Isabell-Alissandra Melzer-Pellmann*†}

Deutsches Elektronen-Synchrotron DESY

E-mail: isabell.melzer@desy.de

The current status of searches for supersymmetry at the CERN LHC, conducted by the ATLAS and CMS experiments, is presented, with focus on the latest results of searches for the supersymmetric partners of the quarks and gluons, the gauge bosons and the leptons.

European Physical Society Conference on High Energy Physics - EPS-HEP2019 -

10-17 July, 2019

Ghent, Belgium

* Speaker.

${ }^{\dagger}$ On behalf of the ATLAS and CMS Collaborations 


\section{Introduction}

Supersymmetry (SUSY) is a consistent theory, which assigns to each half-integer spin particle a partner particle with integer spin and vice versa. SUSY offers solutions to several open questions in particle physics. In $R$-parity conserving models, the lightest SUSY particle (LSP) is stable and as a neutral and weakly interacting particle a viable dark matter candidate. Furthermore, it cancels the large corrections by fermion loops, especially of the top quarks, to the Higgs boson mass with the contributions of the boson partners. It also allows for the unification of the forces at about $10^{16} \mathrm{GeV}$. In addition, neutrinos are expected to have masses in SUSY, and the incorporation of gravity is possible as well.

Searches for SUSY particles are driven by the their cross sections, which are higher for the strongly produced particles, the partners of the gluon (gluino, $\widetilde{\mathrm{g}}$ ) and quark (squark, $\widetilde{\mathrm{q}}$ ), than for the electroweak produced particles. The SUSY partners of the photon, Z, W, and Higgs boson mix to four neutral (neutralinos, $\widetilde{\chi}_{1}^{0}$ to $\widetilde{\chi}_{4}^{0}$ ) and four charged (charginos, $\widetilde{\chi}_{1}^{ \pm}$and $\widetilde{\chi}_{2}^{ \pm}$) mass eigenstates. Depending on their mixing, they are also referred to as higgsinos, binos, or winos. The smallest cross sections are given for the partners of the leptons, the sleptons. The searches are performed in a systematic approach to cover all possible production and decay modes, $R$-parity conserving as well as $R$-parity violating models, and also dedicated searches targeting special scenarios, e.g. compressed spectra in which the SUSY particles have only small mass differences leading to soft final-state particles, and long-lived particles.

These proceedings focus mainly on the latest results of SUSY searches by the ATLAS [1] and CMS [2] Collaborations, with data taken at the CERN LHC [3] at a center-of-mass energy of $13 \mathrm{TeV}$. The results are interpreted in terms of simplified models, where it is assumed that only one heavier SUSY particle and the LSP can be produced at the LHC, and the other partner particles have higher masses. In some models also one or two intermediate-mass SUSY particles are assumed to be produced in the decay of the heavier SUSY particle.

\section{Searches for squarks and gluinos}

Typical scenarios for the decay of the strongly interacting SUSY particles, squarks and gluinos, lead to signatures with large hadronic activity $\left(H_{\mathrm{T}}\right)$ due to the heavy squarks or gluinos, and large missing transverse momentum ( $p_{\mathrm{T}}^{\text {miss }}$ ) because of the LSPs. In addition to requirements on these two variables, searches for strong SUSY production require a large number of jets, and possibly jets that are tagged to originate from $b$ quarks based on lifetime and secondary vertex requirements. Such searches are often binned in the main search variables, in order to be sensitive to a large number of new-physics scenarios. In the following, a generic search in the full-hadronic final state, which is binned in the magnitude of the vector $p_{\mathrm{T}}$ sum of the jets $\left(H_{\mathrm{T}}^{\mathrm{miss}}\right), H_{\mathrm{T}}$, the total number of jets $\left(N_{\text {jet }}\right)$, and the number of tagged b jets $\left(N_{\mathrm{b}-\text { jet }}\right)$ will be discussed. The main backgrounds are caused by events which contain a genuine lepton that is not identified or out of acceptance (mainly $\mathrm{W}+\mathrm{jets}$ and $\mathrm{t} \overline{\mathrm{t}}$ events), irreducible background with genuine $p_{\mathrm{T}}^{\text {miss }}$, and quantum chromodynamics (QCD) multijet events, where significant $H_{\mathrm{T}}^{\text {miss }}$ can arise if the $p_{\mathrm{T}}$ of a jet is mismeasured, out of acceptance, or contains neutrinos (in case of b jets). All three backgrounds are determined in control regions in data, e.g. the background from single-lepton events is determined in a single- 
lepton control region, while the irreducible background with genuine $p_{\mathrm{T}}^{\text {miss }}$, mainly due to $\mathrm{Z} \rightarrow v v$ events, is determined in a $\gamma+$ jets and a $Z \rightarrow \ell \ell$ control sample, where $\ell$ refers to either an electron or a muon.

Figure 2 (left) shows the background prediction for each of the signal bins in comparison with the data. Since no significant excess of data over the standard model (SM) prediction is observed, limits are set in terms of several simplified models of gluino and squark pair production. Figure 2 (right) contains the limit for the decay of the squark to a quark-antiquark pair of the first and second generation for two different cases: either SUSY partners of all first and second generation squarks are light enough to be produced, or only one squark is light enough to be produced at the LHC. The limits on the masses of directly produced squarks range from 1130 for one light squark to $1630 \mathrm{GeV}$, assuming the prediction of all (mass-degenerate) light-flavored squarks [4].
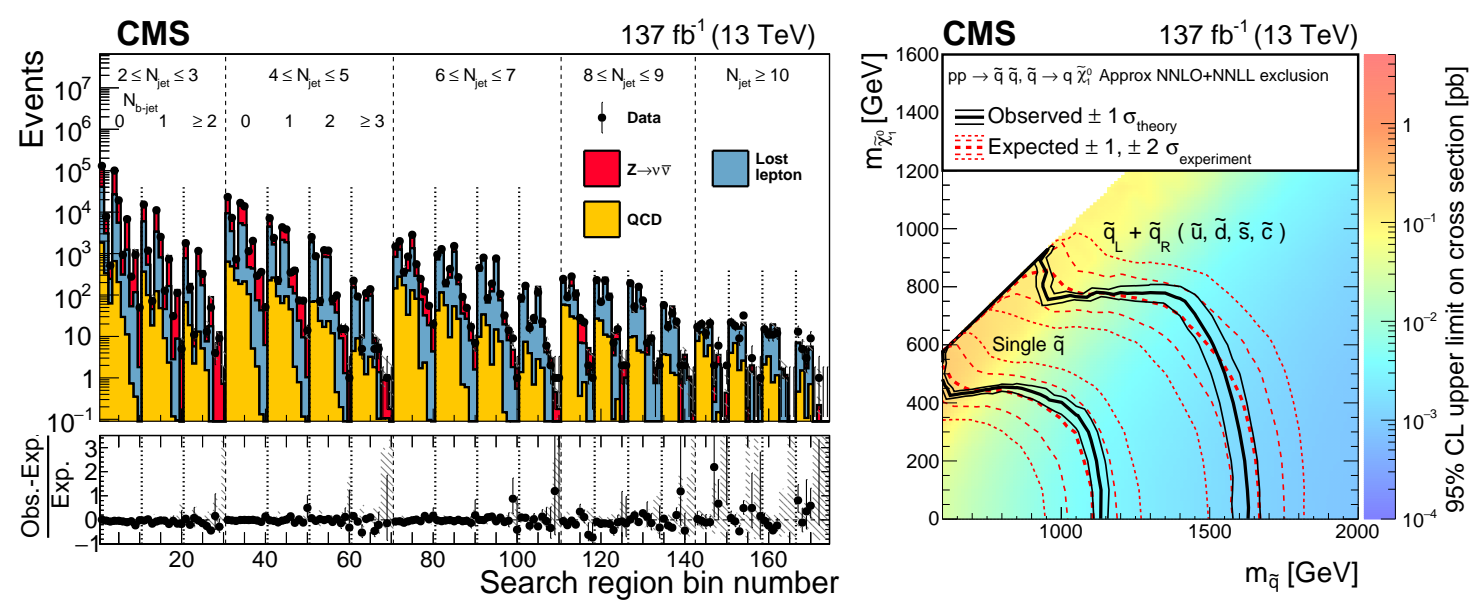

Figure 1: Left: The observed numbers of events and pre-fit SM background predictions in the 174 search bins. The 10 results ( 8 for $N_{\text {jet }} \geq 8$ ) within each region delineated by vertical dashed lines correspond sequentially to 10 (8) kinematic intervals in $H_{\mathrm{T}}$ and $H_{\mathrm{T}}^{\text {miss }}$. Right: The 95\% CL upper limits on the production cross sections of the squark pair production, where each squark decays to a quark and the LSP. The thick black (solid) curves show the observed exclusion limits, the thin black (solid) curves show the changes in these limits as the signal cross sections are varied by their theoretical uncertainties, the thick red (dashed) curves present the expected limits under the background-only hypothesis, and the thin red (dotted) curves indicate the variation of the experimental uncertainties by one standard deviation [4].

Figure 2 shows the limit for the cases where each gluino decays into a top quark-antiquark pair and the LSP and for the decays to the first and second generation squarks. Gluinos with masses below $2050 \mathrm{GeV}$ are excluded at $95 \%$ confidence level for $\widetilde{\mathrm{g}} \rightarrow \mathrm{t} \tilde{\mathrm{\chi}} \widetilde{\chi}_{1}^{0}$, while the limit is slightly weaker for the gluino decay to the first two generation squarks, where masses below $2 \mathrm{TeV}$ are excluded [5].

Besides the generic searches, the experiments have also performed more specialized searches to target specific models like $R$-parity violating decays or longer decay chains that might involve decays to $\mathrm{W}$ or $\mathrm{Z}$ bosons. Such searches can profit from leptonic decay channels and specifically tuned signal regions. For example, by requiring two same-sign leptons the SM background is re- 

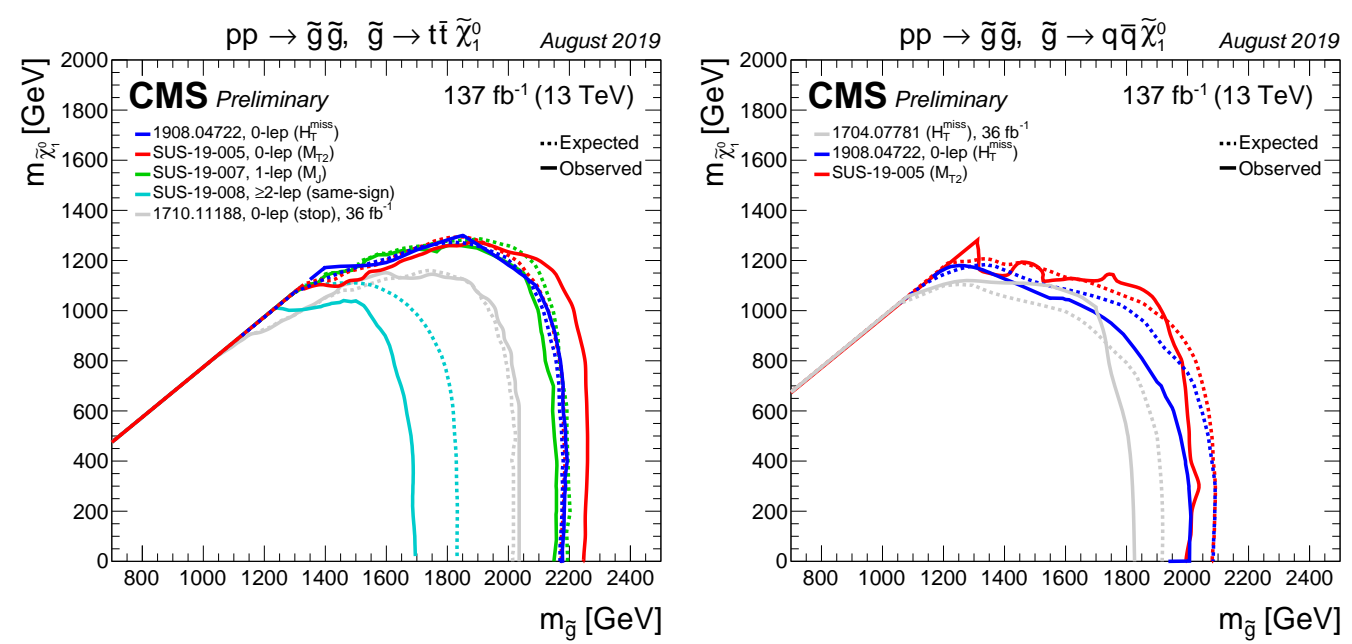

Figure 2: Left: The 95\% CL upper limits on the production cross sections of gluino pair production with decay of the gluino into a top quark-antiquark pair and the LSP. Right: Corresponding upper limits for the decay of the gluino into a first or second generation quark-antiquark pair and the LSP for different analyses $[4,5,6,7,8,9]$.

duced drastically and backgrounds that are minor in all hadronic searches become critical, like $\mathrm{WZ}+$ jets in signal regions with zero b-tagged jets and $\mathrm{t}+\mathrm{t}$ in signal regions with $\mathrm{b}$-jets, which are both estimated from simulation normalized to the theory cross section, weighted by several scale factors, e.g. to correct for the mismodelling of inefficiencies associated with the lepton reconstruction and identification and the trigger. Background from events with charge-flipped electron (mainly $t \bar{t}$ and $\mathrm{Z}+\mathrm{jets}$ events) are estimated from data by weighting opposite-sign dilepton events by the flip rate. Also misidentified and non-prompt leptons are estimated from data. Signal regions are defined based on the number of jets, b-tagged jets, $p_{\mathrm{T}}^{\text {miss }}$, and the effective mass ( $\left.m_{\mathrm{eff}}\right)$. Gluino pair production with gluino decay to a pair of light quarks and a longer decay chain including a $\widetilde{\chi}_{1}^{ \pm}$, that further decays to a $\mathrm{W}$ boson and a $\widetilde{\chi}_{2}^{0}$, which then decays into a $\mathrm{Z}$ boson and the $\widetilde{\chi}_{1}^{0}$, is excluded for gluino masses below $1.6 \mathrm{TeV}$ at $95 \%$ confidence level. Gluino pair production with the $R$-parity violating decay of the gluino into a top, a bottom and a down quark is also excluded for gluino masses below 1.6 TeV [10].

Dedicated searches are performed for top squark pair production. Depending on the mass difference $\Delta m$ between the top squark $\widetilde{\mathrm{t}}_{1}$, the lighter of two mass eigenstates of the mixing of the SUSY partners of the left- and right-handed top quark) and the LSP, assumed to be the $\widetilde{\chi}_{1}^{0}$, different decays are possible:

- $\Delta m \leq m_{\mathrm{W}}+m_{\mathrm{b}}$ : If the mass difference is smaller than the mass of the $\mathrm{W}$ boson and the $\mathrm{b}$ quark, only decays into the charm quark and the LSP or decays into the b quark, a fermionantifermion pair, and the LSP are possible.

- $m_{\mathrm{W}}+m_{\mathrm{b}} \leq \Delta m \leq m_{\mathrm{t}}$ : In this case the top squark decays via a three-body process into a $\mathrm{b}$ quark, a W boson and the LSP.

- $\Delta m>m_{\mathrm{t}}$ : Here the top squark decays into a top quark and the LSP. 
Different analysis strategies are necessary to tackle these different decay modes. Updates with the full Run 2 luminosity have been performed in the single-lepton final state for $m_{\mathrm{W}}+m_{\mathrm{b}} \leq$ $\Delta m \leq m_{\mathrm{t}}$ exploiting advanced neural networks to improve the selection efficiency, such that top squark masses up to $720 \mathrm{GeV}$ with neutralino masses up to $580 \mathrm{GeV}$ are excluded [11], as shown in Fig. 3 (left). The analysis targeting mainly $\Delta m>m_{\mathrm{t}}$ includes special features to enhance the sensitivity in the corridor with $\Delta m \approx m_{\mathrm{W}}$ by developing soft b-tagging. In the region with $\Delta m \approx m_{\mathrm{W}}$ the analysis is based on tagging of an initial state radiation (ISR) jet and a soft lepton. Resolved and boosted top tagging enhances the sensitivity in the high stop mass region. Top squark masses up to $1.2 \mathrm{TeV}$ are set for a nearly massless LSP. For models with top squark masses of $1 \mathrm{TeV}$, neutralino masses up to $600 \mathrm{GeV}$ are excluded [12], as shown in Fig. 3 (right).
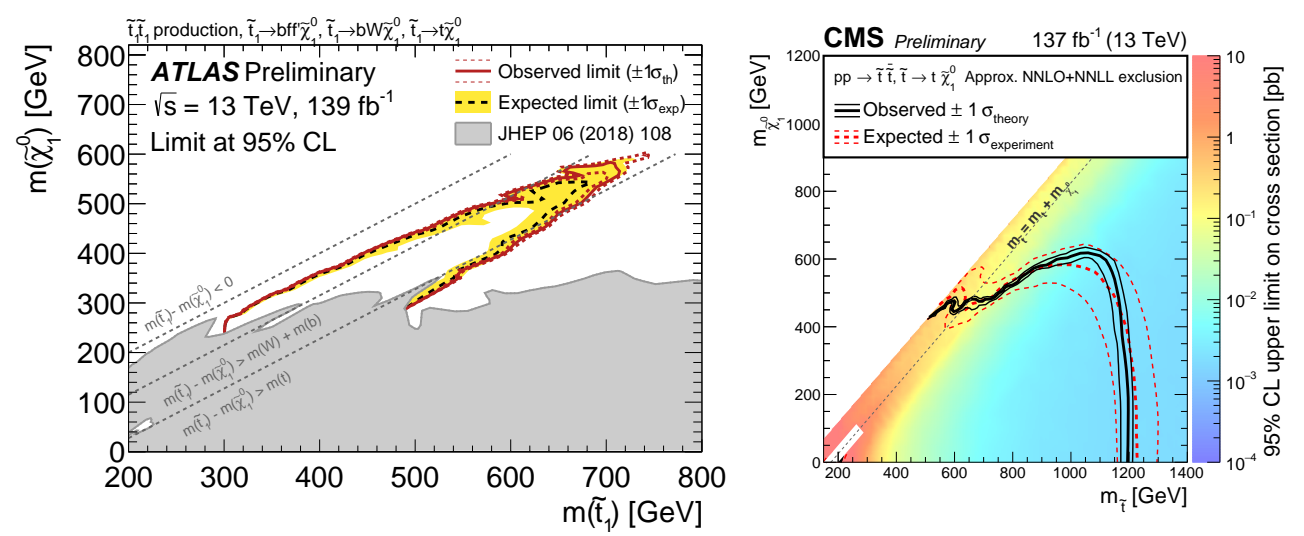

Figure 3: Left: Exclusion limits for direct top squark pair production in an analysis targeting the three-body decay of the top squark into a b quark, a W boson and the LSP [11]. Right: Exclusion limits for direct top squark pair production targeting mainly the decay into a top quark and the LSP [12].

\section{Searches for neutralinos and charginos}

The mixing of the wino, bino and higgsino to their mass eigenstates, the neutralinos and charginos, is governed by their gauge eigenstate mass terms $M_{1}, M_{2}$ and $\mu$. Furthermore, the size of the mass splitting between them is governed by their mass ordering, as shown in Fig. 4. In the case $M_{1}<M_{2}<\mu$, the $\widetilde{\chi}_{1}^{0}$ is bino-like, and the $\widetilde{\chi}_{2}^{0}$ and $\widetilde{\chi}_{1}^{ \pm}$are wino-like and have small mass differences, while the mass difference to the $\widetilde{\chi}_{1}^{0}$ is usually larger, allowing for signatures with $p_{\mathrm{T}}^{\text {miss }}$ and possibly leptons or jets from Z-, W- or h-boson decays. On the contrary, for the mass hierarchy $\mu<M_{2}<M_{1}$, the $\widetilde{\chi}_{1}^{0}$ as well as $\widetilde{\chi}_{2}^{0}$ and $\widetilde{\chi}_{1}^{ \pm}$are higgsino-like and are almost mass degenerate, leading to soft final states which is experimentally challenging, and in the case of very small mass differences, also to final states with long-lived particles.

A search for neutralino-chargino production in the bino-wino scenario is done in the singlelepton final state [13], assuming that the $\widetilde{\chi}_{1}^{ \pm}$decays to a W boson and the LSP, and the $\widetilde{\chi}_{2}^{0}$ decays to a Higgs boson and the LSP. The latter decay is dominant for many parameter choices, if the mass-splitting between the two lightest neutralinos is larger than the Higgs boson mass and the higgsinos are heavier than the winos. This analysis selects leptonic W bosons and Higgs decays 


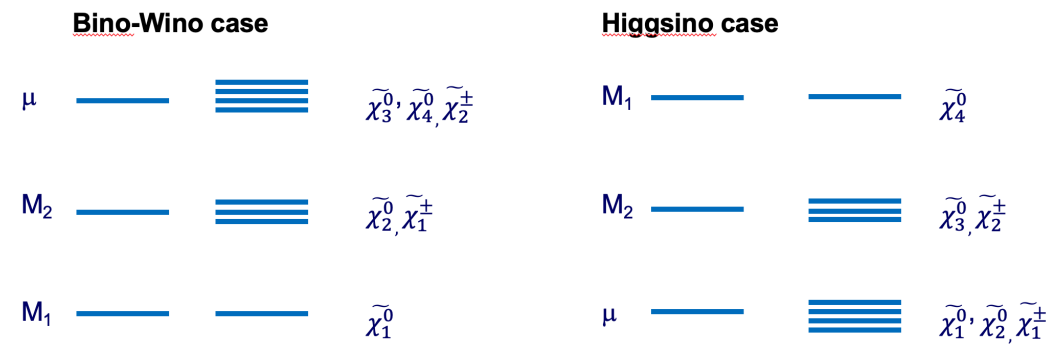

Figure 4: Mass splitting of the SUSY partners of the gauge bosons for the different mass hierarchy scenarios.

to two b quarks. The main backgrounds in this search are $\mathrm{tt}, \mathrm{tW}$, and $\mathrm{W}+\mathrm{jets}$ events, which are normalized in dedicated control regions. The performance of this normalization is tested in several dedicated validation regions. The comparison of the observed and expected event yields in control, validation, exclusion, and discovery signal regions is given in Fig. 5 (left). Since no significant excess is observed, limits on the production of the $\widetilde{\chi}_{1}^{ \pm}$and the $\widetilde{\chi}_{2}^{0}$ are calculated and given in Fig. 5 (right). Masses of $\widetilde{\chi}_{2}^{0}$ and $\widetilde{\chi}_{1}^{ \pm}$up to $740 \mathrm{GeV}$ are excluded at $95 \%$ confidence level for a massless $\widetilde{\chi}_{1}^{0}$.
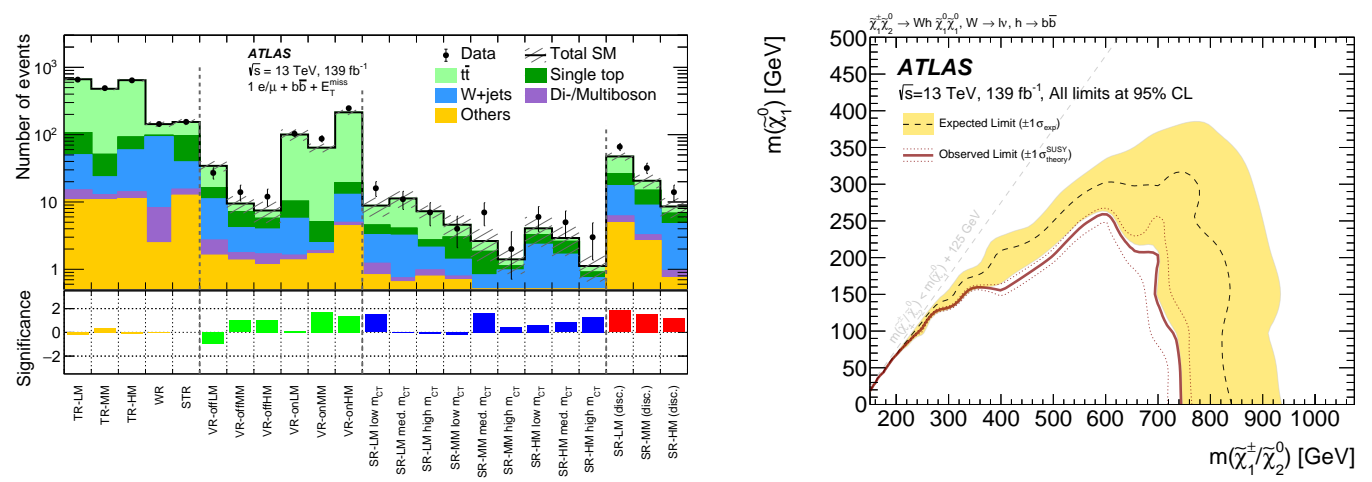

Figure 5: Left: Comparison of the observed and expected event yields in control, validation, exclusion, and discovery signal regions. Uncertainties in the background estimates include both the statistical (in the simulated event yields) and systematic uncertainties. The bottom panel shows the significance of the differences between the observed and expected yields. Not all regions shown here are statistically independent. Right: Model-dependent exclusion contour at 95\% CL on the production of a chargino and a next-to-lightest neutralino. The observed limit is given by the solid line with the signal cross-section uncertainties shown by the dotted lines. Expected limits are given by the dashed line with uncertainties shown by the shaded band [13].

In the case of a higgsino scenario the analysis is more challenging because of the nearly massdegenerate spectrum and a special search strategy has to be employed to gain sensitivity to such scenarios. One possibility in the search for $\widetilde{\chi}_{2}^{0}-\widetilde{\chi}_{1}^{ \pm}$production is the usage of ISR jets to trigger events [14]. Assuming that the $\widetilde{\chi}_{2}^{0}$ decays into a (virtual) $\mathrm{Z}$ boson and the $\widetilde{\chi}_{1}^{0}$ and a $\widetilde{\chi}_{1}^{ \pm}$to a (virtual) $\mathrm{W}$ boson and a $\widetilde{\chi}_{1}^{0}$, the events are required to contain $p_{\mathrm{T}}^{\text {miss }}$, an ISR jet, and two sameflavour, oppositely charged, low transverse momentum leptons. A specialty of this analysis is that 
instead of the second lepton also an isolated low- $p_{\mathrm{T}}$ track is used. Electrons are required to have $p_{\mathrm{T}}>4.5 \mathrm{GeV}$ or a matched track with $p_{\mathrm{T}}>1 \mathrm{GeV}$, or muons with $p_{\mathrm{T}}>3 \mathrm{GeV}$ or matched track with $p_{\mathrm{T}}>2 \mathrm{GeV}$, which extends the sensitivity to invariant masses of the lepton and the track $\left(m_{\ell \text { track }}\right)$ to $1 \mathrm{GeV}$ where most of the signal is expected, as shown in Fig. 6 (left). The reducible background from fake and non-prompt leptons (mainly from heavy flavor decay) is determined from data, while the irreducible background with two prompt leptons ( $\mathrm{t} t, \mathrm{tW}, \mathrm{WW}, \mathrm{WZ}, \mathrm{Z} \rightarrow \tau \tau$ ) is determined from simulation normalized to data in dedicated control regions. Since no excess of data over the SM prediction is observed, limits are determined as shown in Fig. 6 (right). Higgsinos are excluded with masses below $162 \mathrm{GeV}$ at a mass splitting of $10 \mathrm{GeV}$, and extending down to a mass splitting of $2.6 \mathrm{GeV}$ at the LEP limit.
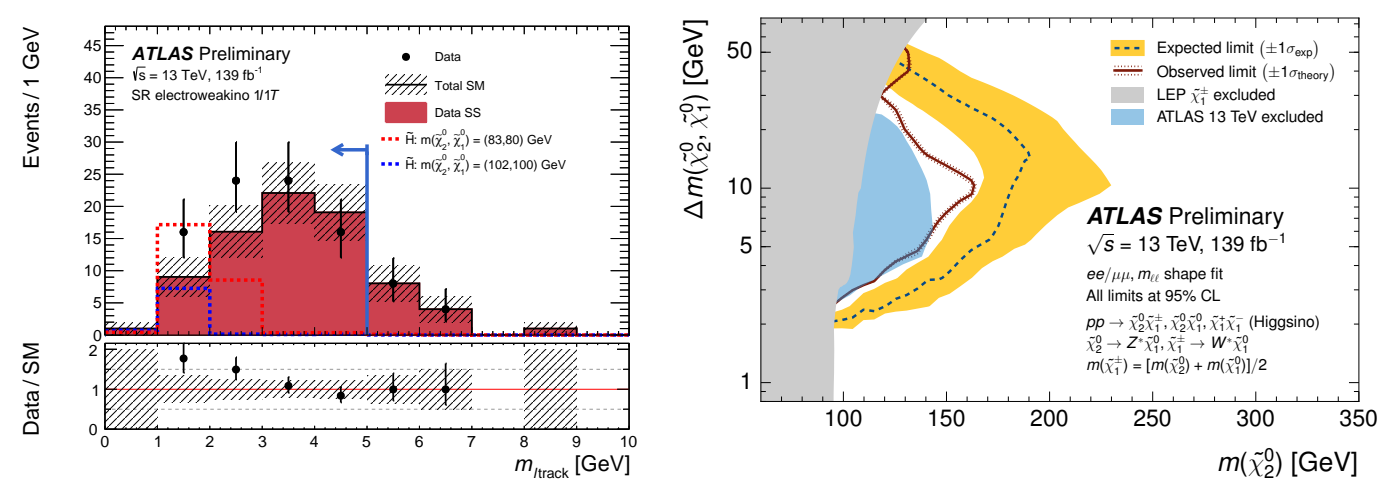

Figure 6: Left: Invariant mass of the lepton and the isolated low- $p_{\mathrm{T}}$ track. Right: Expected 95\% CL exclusion sensitivity (blue dashed line) with $\pm 1 \sigma_{\text {exp }}$ (yellow band) from experimental systematic uncertainties and observed limits (red solid line) with $\pm 1 \sigma_{\text {theory }}$ (dotted red line) from signal cross section uncertainties for simplified models of direct Higgsino production [14].

\section{Searches for sleptons}

Searches for sleptons are challenging because of the very small cross section at the LHC, compared to other SUSY particles of similar mass. An analysis based on the full Run 2 dataset assumes slepton pair production with mass-degenerate sleptons of all three generations, and decay of each slepton to a lepton and the $\widetilde{\chi}_{1}^{0}$. The analysis requires the presence of exactly two isolated electrons or muons with opposite electric charge, and significant $p_{\mathrm{T}}^{\text {miss }}$. The main discriminating variable of this search is the stransverse mass $m_{\mathrm{T} 2}$, which is a kinematic variable used to bound the masses of a pair of particles that are presumed to have each decayed into one visible and one invisible particle $[15,16]$. The main irreducible backgrounds, WZ, WW, ZZ, and t⿱t production, are estimated using simulations, and are normalised using a simultaneous likelihood fit to data in dedicated control regions. The main reducible background consists of fake non-prompt leptons and is estimated from data. As shown in Fig. 7 (left), slepton masses up to $700 \mathrm{GeV}$ can be excluded [17].

SUSY models where the $\widetilde{\tau}$ is lighter than the other sleptons are especially well motivated for cases where the $\tilde{\tau}$ is the next-to-lightest SUSY particle, since $\tilde{\tau}$-neutralino coannihilation in the early universe can accommodate the observed DM relic density. The existence of a light $\tilde{\tau}$ would 
enhance the rate of production of final states with $\tau$ leptons in collider experiments. Different mixing scenarios of the SUSY partners of the left- and right-handed $\tau$ leptons are possible, e.g. only the partner of the left-handed $\tau$ lepton $\left(\widetilde{\tau}_{L}\right)$ could be produced, or both, $\widetilde{\tau}_{L}$ and $\widetilde{\tau}_{R}$, could be mass degenerate, which would enhance the sensitivity due to larger production rate. The ATLAS Collaboration has already analyzed the full Run 2 dataset in the full-hadronic final state [18], where the main background are events where jets are misidentified as $\tau$ leptons, QCD multijet events, which are determined from data, and $\mathrm{W}+\mathrm{jets}$ events, from which the shape is taken from simulation, normalized in a control region in data. No excess of data over simulation is observed, and $\widetilde{\tau}_{L}$ pair production is excluded for the first time at the LHC, as shown in Fig. 7, for $\widetilde{\tau}_{L}$ masses between 160 and $300 \mathrm{GeV}$. For the mass-degenerate case, masses between 120 and $390 \mathrm{GeV}$ are excluded. The CMS Collaboration has analyzed only 2016 and 2017 data, but combined the all-hadronic and semileptonic final states [19], and thereby excludes the gap between the former LEP exclusion limit of $90 \mathrm{GeV}$ and $120 \mathrm{GeV}$ from the ATLAS result.
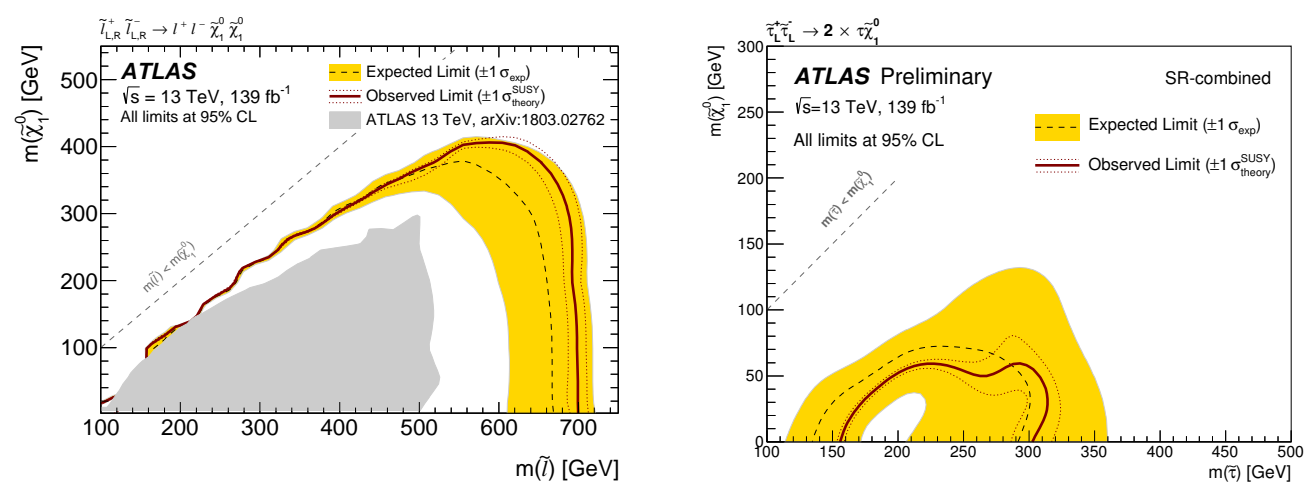

Figure 7: Left: Limit on slepton pair production with mass-degenerate sleptons of all three generations [17]. Right: Limit on $\tilde{\tau}$ pair production, assuming that only the SUSY partner of the left-handed $\tau$ lepton is produced. The observed limit is given by the solid line with the signal cross-section uncertainties shown by the dotted lines. Expected limits are given by the dashed line with uncertainties shown by the shaded band [18].

\section{Searches for long-lived particles}

Apart from the LSP other particles could be long-lived as well, caused e.g. by:

- Very weak $\widetilde{\chi}_{1}^{0}$-gravitino coupling, leading e.g. to delayed photons or $\mathrm{Z}$ bosons.

- $R$-parity violating (RPV) SUSY, where the sparticle lifetime is proportional to the RPV coupling, possibly leading to a displaced vertex if the coupling is of the order of $\mathscr{O}\left(10^{-5}\right)$.

- Small mass difference, e.g. $\Delta M\left(\widetilde{\chi}_{1}^{ \pm}-\widetilde{\chi}_{1}^{0}\right) \approx 100 \mathrm{MeV}$, leading e.g. to soft pion emission, where the pion is not reconstructed, causing a disappearing track signature.

Delayed photons are expected in gauge-mediated SUSY breaking (GMSB), where there is a weak coupling of the $\widetilde{\chi}_{1}^{0}$ to the photon and the gravitino $(\widetilde{G})$. A search with 2016 and 2017 data [20] 
is based on impact angles pointing significantly away from the primary interaction vertex and delayed arrival times at the ECAL of the order of nanoseconds. The investigated models assume squark or gluino pair production and decay to squarks and the $\widetilde{\chi}_{1}^{0}$, which then further decays to a photon or a $\mathrm{Z}$ boson. Squark decays into a $\widetilde{\chi}_{1}^{ \pm}$and a quark, where the $\widetilde{\chi}_{1}^{ \pm}$decays to a $\widetilde{G}$ and a $\mathrm{W}$ boson, are also taken into account. For $\widetilde{\chi}_{1}^{0}$ proper decay lengths of $10^{1}, 10^{2}, 10^{3}$, and $10^{4} \mathrm{~cm}$, masses up to $320,525,360$, and $215 \mathrm{GeV}$ are excluded at $95 \%$ confidence level, respectively, as shown in Fig. 8 (left). Compared to earlier searches, the requirement of a single photon increases the sensitivity to long lifetimes.

Assuming $R$-parity violation (RPV), the $\widetilde{\chi}_{1}^{0}$ can possibly decay into two charged leptons and a neutrino through a weak RPV coupling, which might lead to a lifetime of picoseconds to nanoseconds. In this case the decay may be observed as a displaced vertex in the inner tracking volume. A dedicated analysis targets models in which the $\widetilde{\chi}_{1}^{0}$, produced via squark-antisquark production, decays into $\ell^{+} \ell^{\prime}$, with $(\ell, \ell=\mathrm{e}, \mu)$ with a finite lifetime, leading to a measurable displaced vertex [21]. This analysis depends on special techniques like the development of a special trigger on muon chamber tracks and photons and special large-radius tracking and displaced vertex reconstruction. The main backgrounds are cosmic-ray muons and random crossing of two uncorrelated leptons. Cross section limits are calculated for specific squark and neutralino masses. For a $700 \mathrm{GeV}$ squark, neutralinos with masses of $50-500 \mathrm{GeV}$ and mean proper lifetimes between $1 \mathrm{~mm}$ to $6 \mathrm{~m}$ are excluded. For a 1.6 TeV squark, shown in Fig. 8 (right), $c \tau$ values between $3 \mathrm{~mm}$ to $1 \mathrm{~m}$ are excluded for $1.3 \mathrm{TeV}$ neutralinos.
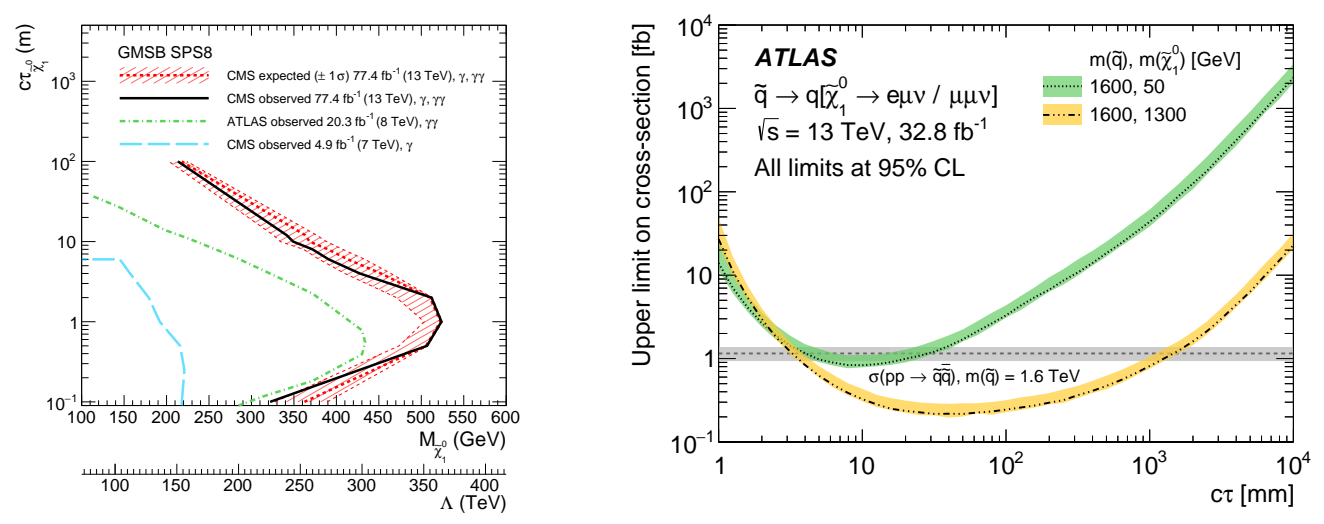

Figure 8: Left: The 95\% CL exclusion contours for the GMSB neutralino production cross section, shown as functions of the neutralino mass, or equivalently the SUSY breaking scale, $\Lambda$, for typical GMSB model parameters, and the neutralino proper decay length [20]. Right: Observed upper limits at 95\% CL on the squark-antisquark production cross-section as a function of the mean proper lifetime of the $\widetilde{\chi}_{1}^{0}$, in units of $c \tau$, for the $\lambda_{121}$ scenario and a $1.6 \mathrm{TeV}$ squark. The horizontal lines indicate the theoretical (approx. NNLO+NNLL) cross-sections with the uncertainties shown as shaded bands. The shaded bands around the observed limits indicate the $1 \sigma$ variations in the expected limit [21]. 


\section{Summary and outlook}

The ATLAS and CMS experiments have already performed a number of searches for supersymmetry with full Run 2 luminosity, where the searches cover not only broad signatures tested with simplified models, but also tackle specific signatures like long-lived particles. While more results based on the full Run 2 luminosity are to come in the next months, the experiments are already in preparation of Run 3. In a few years, the High Luminosity-LHC will start to deliver up to five times higher luminosity, leading to an expected integrated luminosity of $3000-4000 \mathrm{fb}^{-1}$. With these data it will be possible to cover rare processes, like direct $\tilde{\tau}$ pair production and higgsino-like neutralino and chargino production [22, 23], shown in Fig. 9.
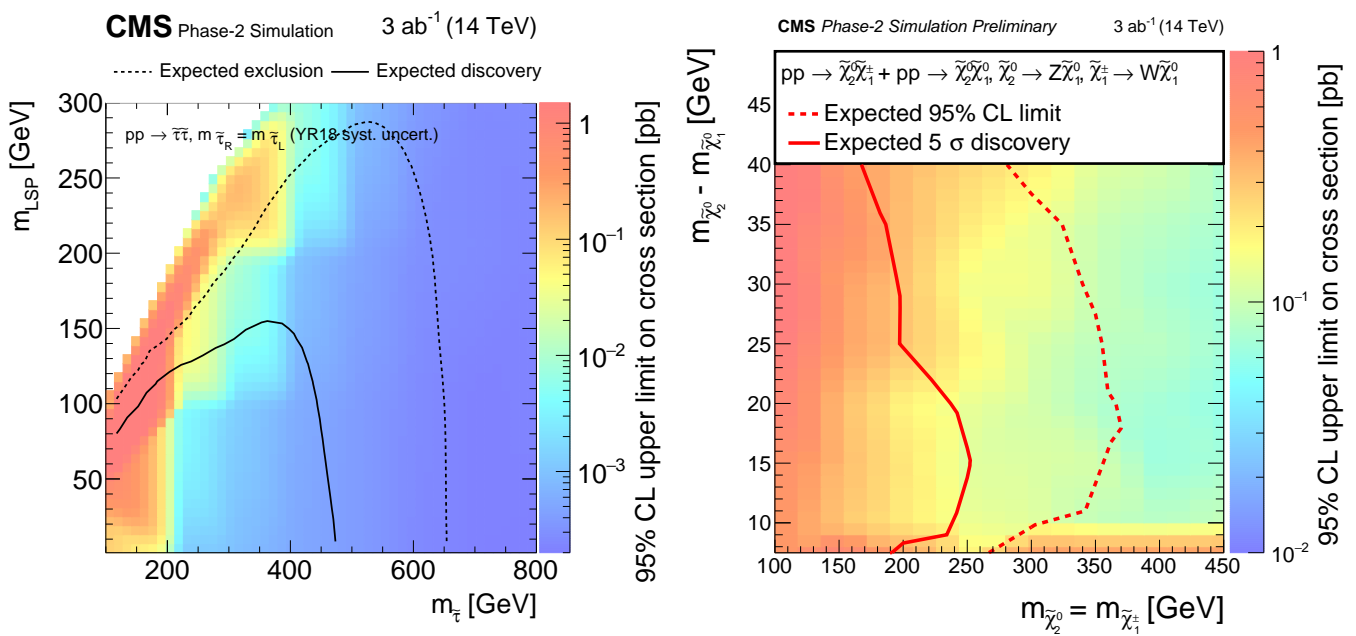

Figure 9: Left: The expected upper limits at the $95 \% \mathrm{CL}$ and the $5 \sigma$ discovery potential for direct $\widetilde{\tau}$ production analyzed with combination of the results of the full-hadronic and semileptonic channels [22]. Right: The $5 \sigma$ discovery contours and expected $95 \% \mathrm{CL}$ exclusion contours for light higgsino-like neutralino and chargino production. Results are presented for $\Delta M_{\widetilde{\chi}_{1}^{ \pm}, \widetilde{\chi}_{1}^{0}}>7.5 \mathrm{GeV}$ [23].

\section{References}

[1] ATLAS Collaboration, JINST 3 (2008) S08003.

[2] CMS Collaboration, JINST 3 (2008) S08004.

[3] L. Evans and P. Bryant (editors), JINST 3 (2008) S08001.

[4] CMS Collaboration, arXiv:1908.04722 [hep-ex].

[5] CMS Collaboration, arXiv:1909.03460 [hep-ex].

[6] CMS Collaboration, CMS-PAS-SUS-19-007 (2019), https://cds.cern.ch/record/2685097.

[7] CMS Collaboration, CMS-PAS-SUS-19-008 (2019), https://cds.cern.ch/record/2685097.

[8] CMS Collaboration, Phys. Rev. D 97, 012007 (2018).

[9] CMS Collaboration, Phys. Rev. D 96, 032003 (2017). 
[10] ATLAS Collaboration, arXiv:1909.08457 [hep-ex].

[11] ATLAS Collaboration, ATLAS-CONF-2019-017 (2019), http://cdsweb.cern.ch/record/2676594.

[12] CMS Collaboration, CMS-PAS-SUS-19-009 (2019), https://cds.cern.ch/record/2682157.

[13] ATLAS Collaboration, arXiv:1909.09226.

[14] ATLAS Collaboration, ATLAS-CONF-2019-014 (2019), https://cds.cern.ch/record/2675954.

[15] C.G. Lester and D.J. Summers, Phys. Lett. B463(1999) 99.

[16] A. Barr, C.G. Lester and P. Stephens, J. Phys. G29(2003) 2343.

[17] ATLAS Collaboration, arXiv:1908.08215.

[18] ATLAS Collaboration, ATLAS-CONF-2019-018 (2019), https://cds.cern.ch/record/2676595.

[19] CMS Collaboration, arXiv:1907.13179.

[20] CMS Collaboration, arXiv:1909.06166.

[21] ATLAS Collaboration, arXiv:1907.10037.

[22] CMS Collaboration, CMS-PAS-FTR-18-010 (2018), https://cds.cern.ch/record/2647985.

[23] CMS Collaboration, CMS-PAS-FTR-18-001 (2018), https://cds.cern.ch/record/2648538. 